$$
1995119848
$$

\section{SENSITIVITY ANALYSIS FOR THE DYNAMIC RESPONSE OF VISCOPLASTIC SHELLS OF REVOLUTION}

\author{
Makarand Kulkarnl and Ahmed K. Noor \\ Center for Computational Structures Technology \\ NASA Langley Research Center \\ University of Virginla \\ Hampton, Virginia
}

$55-39$

ABS. ONLY

47069

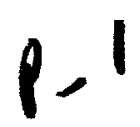

\begin{abstract}
A computational procedure is presented for evaluating the sensitivity coefficients of the dy namic axisymmetric response of viscoplastic shells of revolution. The analytical fomulation is based on Reissner's large deformation shell theory with the effects of transverse shear deformation, rotatory inertia and moments turning around the normal to the middle surface included. The material model is chosen to be isothermal viscoplasticity, and an associated how rule is used with a von Mises effective stress. A mixed formulation is used with the fundamental unknowns consisting of six stress resultants, three generalized displacements and three velocity components.

Spatial discretization is performed using finite elements, will disconlinuous stress resultants across element interfaces. The temporal integration is performed by using an explicit central difference scheme (leap-frog method) with an implicit constitutive update. The sensitivity coefficients are evaluated using a direct differentiation approach. Numerical results are presented for a spherical cap subjected to step loading, and a circular plate subjected to impulsive loading. The sensitivity coefficients are generated by evaluating the derivatives of the response quantities with respect to the thickness, mass density, Young's nodulus, and two of the material parameters characterizing the viscoplastic response. Time histor ies of the response and sensitivity coefficients are presented, along with spatial distributions of these quantities at selected times.
\end{abstract}

\title{
NOMENCLATURE
}

$\mathbf{a}$

IC

$\mathrm{E}$

[F]

(rex)

\{fint $\}$

$\{\mathrm{G}(\langle\mathrm{X}\rangle)\}$ radius of circular plate

characteristic strain rate

matrix of material stiffuess coefficients, see Eq. B 14, Appendix B

Young's noxdulus

global linear flexibility matrix of the shell

vector of nodal external forces

vector of nodal internal forces

vector of nonlinear contributions 